\title{
Codes for Differential Signaling with Many Antennas
}

\author{
Babak Hassibi, Bertrand Hochwald, \\ Amin Shokrollahi and Wim Sweldens \\ Mathematical Sciences Center \\ Lucent Technologies \\ 600 Mountain Avenue \\ Murray Hill, NJ 07974 \\ e-mail: \\ \{hassibi, hochwald, mshokrollahi, wim\}@lucent.com
}

\begin{abstract}
We construct signal constellations for differential transmission with multiple basestation antennas. The signals are derived using the theory of fixed-point-free groups and are especially suitable for mobile cellular applications because they do not require the handset to have more than one antenna or to know the time-varying propagation environment. Yet we achieve full transmitter diversity and excellent performance gains over a single-antenna system.
\end{abstract}

\section{INTRODUCTION}

Differential phase-shift keying (DPSK) is a well-known technique for transmitting digital information across an unknown time-varying channel. Let the data consist of a sequence of integers $z_{1}, z_{2}, \ldots \in$ $\{0, \ldots, L-1\}$ where $L$ is the size of our alphabet (often a power of two). DPSK with a single transmitter transmits complex baseband signals that obey the recursion

$$
s_{t}=v_{z_{t}} s_{t-1} \quad t=1,2, \ldots,
$$

where $s_{0}=1$ and the $v_{z_{t}} \in\left\{1, e^{2 \pi i / L}, \ldots, e^{2 \pi i(L-1) / L}\right\}$ are $L$ points around the complex unit circle.

We can extend this differential scheme to $M>1$ transmit antennas by transmitting $M \times M$ matrices that obey the recursion

$$
S_{\tau}=V_{z_{\tau}} S_{\tau-1} \quad \tau=1,2, \ldots,
$$

where $S_{0}$ is the $M \times M$ identity matrix and $V_{z_{\tau}}$ are complex unitary data matrices [1]. (See also [3] for a similar differential scheme and [2] for a differential scheme based on orthogonal designs.) Each row of the transmission matrix $S_{\tau}$ specifies what is transmitted on the $M$ transmit antennas; hence each $S_{\tau}$ specifies what the $M$ antennas do for $M$ time samples. The index $\tau$ marks each block of $M$ time samples.

To use this method effectively, we need to design $L=2^{R M}$ unitary data matrices $\mathcal{V}=\left\{V_{0}, \ldots, V_{L-1}\right\}$, where $R$ is the data rate. We also need a simple decoding algorithm at the receiver. It can be shown that across an unknown flat-fading channel, the received signals on $N$ antennas for $M$ units of time obey the recursion

$$
X_{\tau}=V_{z_{\tau}} X_{\tau-1}+W_{\tau},
$$

where $W_{\tau}$ is a $M \times N$ matrix of additive independent complexGaussian noise. Maximum likelihood decoding simply chooses the $V$-matrix that minimizes the Frobenius norm of the difference between $X_{\tau}$ and $V \cdot X_{\tau-1}$

Unlike with DPSK, the choice of effective unitary data matrices $V_{z_{t}}$ is nontrivial. At high SNR, the performance of a constellation

\begin{tabular}{|c|c|c|c|c|}
\hline & Group type & $L$ & $M$ & Comments \\
\hline 1. & $G_{m, r}$ & $m n$ & $n$ & \\
\hline 2. & $D_{m, r, \ell}$ & $2 m n$ & $2 n$ & Contains quaternions \\
\hline 3. & $E_{m, r}$ & $8 m n$ & $2 n$ & \\
\hline \multirow[t]{3}{*}{4.} & $F_{m, r, \ell}$ & $16 m n$ & $4 n$ & if $n>1$ or \\
\hline & & & & $\ell \not \equiv 1 \bmod m / 3$ \\
\hline & $F_{m, 1, \ell}$ & $16 m n$ & 2 & if $\ell \equiv 1 \bmod m / 3$ \\
\hline 5. & $J_{m, r}$ & $120 m n$ & $2 n$ & \\
\hline 6. & $K_{m, r, \ell}$ & $240 m n$ & $4 n$ & \\
\hline
\end{tabular}
can be shown to depend on

$$
\zeta_{\mathcal{V}}=\frac{1}{2} \min _{\ell^{\prime} \neq \ell}\left|\operatorname{det}\left(V_{\ell^{\prime}}-V_{\ell}\right)\right|^{1 / M}
$$

Table 1: There are six types of groups with $\zeta>0$ : For each group $G, L$ is the order of $G$ (the size of the constellation) and $M$ is the dimension of the representation of $G$ (number of transmit antennas). $n$ is the smallest integer such that $r^{n} \equiv$ $1(\bmod m)$

We wish to choose the constellation $\mathcal{V}$ to have $0 \leqslant \zeta \mathcal{V} \leqslant 1$ as large as possible.

\section{GROUP CONSTELLATIONS}

Equation (1) is the fundamental differential transmission and we see that if the set $\mathcal{V}$ forms a group, the transmitted signal $S_{\tau}$ is always an element of $\mathcal{V}$. In fact, the multiplication in (1) is replaced by a group table-lookup. We therefore ask if there are groups of unitary matrices that have large $\zeta$. Perhaps surprisingly, this question was examined in the early 20th century in the mathematical literature (in a different context!) and answered in large part in [5] using the theory of fixed-point-free groups.

Briefly, a group that has a "representation" in unitary matrices with nonzero $\zeta$ is called fixed-point-free. Zassenhaus in [5] classifies most of the fixed-point-free groups. We complete the classification in [6]. We defer a detailed discussion of these groups to [6] and refer instead to Table 1 where the classes of the groups are outlined. Note that there are only six classes and any group with $\zeta>0$ must fall within one of them. We also list their orders (size of constellation $L$ ) and the dimension of their representations (number of antennas $M$ ).

We see from the table that only the class $G_{m, r}$ gives signal constellations for odd $M$. The class $G_{m, r}$ also contains the diagonal signals corresponding to the cyclic groups studied in [1]. The class $D_{m, r, \ell}$ contains the generalized quaternion (also called dicyclic) 


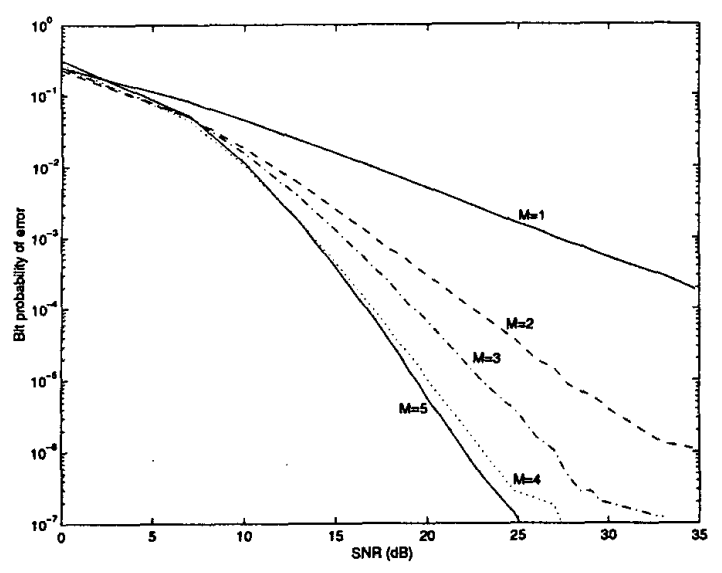

Figure 1: Performance of $M=1,2,3,4$, and 5 transmit antennas and $N=1$ receiver antenna as a function of SNR $\rho$. The channel has unknown Rayleigh fading that is changing continuously according to Jakes' [4] model with parameter $f_{D} T_{s}=0.0025$. The data rate is $R=1$.

groups studied in [3]. Within the classes are many groups that perform very well when simulated on a wireless channel, as can be seen in the next section.

\section{PERformance CURVES}

Figure 1 shows the performance of using cyclic groups (corresponding in Table 1 to $G_{m, 1}$ ) for $M=1, \ldots, 5$ antennas transmitting across a continuously fading channel to one receive antenna with transmission rate $R=1$. (With $M=1$ antenna, standard differential BPSK is used.) The advantages of using more than one antenna are readily seen.

For higher rates, non-Abelian groups seem to provide better performance. As an example of a high-rate fixed-point-free group code, we plot in Figure 2 the performance of the group $F_{15,1,11}$, whose details defy a simple description and appear in [6]. This group has a representation as 240 complex $2 \times 2$ unitary matrices (rate $R=\log (240) / 2 \approx 3.95$ ) suitable for differential transmission over a two-antenna fading channel. We also plot the performance of the best cyclic group with the same rate [1], a $2 \times 2$ orthogonal design [2] (which is not a group) and a generalized quaternion group code [3] with similar rates. All of these codes can also be used with a known channel with a performance gain of approximately $3 \mathrm{~dB}$.

Another example, appearing in Figure 3 , is the group $K_{1,1,-1}$ (again defying a simple description) with a representation in 240 complex $4 \times 4$ matrices (rate $R=\log (240) / 4 \approx 1.98$ ). We also plot the performance of the best cyclic group with the same rate and see that the performance gains are appreciable.

\section{REFERENCES}

[1] B. Hochwald and W. Sweldens, "Differential unitary space-time modulation," to appear in IEEE Trans. Comm. Download available at http: //mars.bel1-labs.com.

[2] V. Tarokh and $H$. Jafarkhani, “A differential detection scheme for transmit diversity," to appear in J. Sel. Area Comm.

[3] B. L. Hughes, "Differential space-time modulation," submitted to IEEE Trans. Info. Theory, 1999.

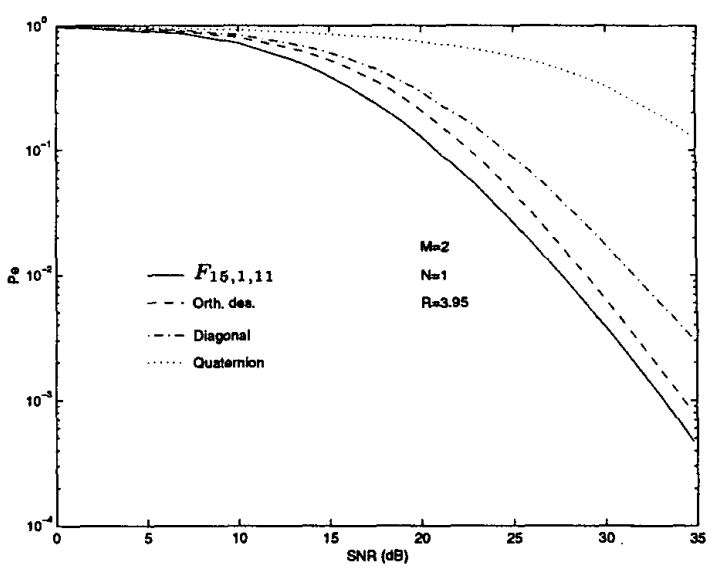

Figure 2: Block-error rate performance of the group $F_{15,1,11}$ for $M=2$ transmitter antennas and $N=1$ receiver antenna. The solid line is $F_{15,1,11}$, which has $L=240$ unitary matrices $(R \approx 3.95$ ). The dashed line is an orthogonal design with 16 th roots of unity $(R=4)$. The dash-dotted line is the best diagonal (Abelian group) construction $(R \approx 3.95$ ). The dotted line is the quaternion group $\left(D_{128,1,-1}\right)$ with $L=256$ matrices $(R=4)$.

[4] W. C. Jakes, Microwave Mobile Communications, Piscataway, NJ: IEEE Press, 1993.

[5] H. Zassenhaus, "Über endliche Fastkörper," Abh. Math. Sem. Hamburg, vol. 11, pp. 187-220, 1936.

[6] B. Hassibi, B. Hochwald, A. Shokrollahi and W. Sweldens, "Representation theory for high-rate multiple-antenna code design," submitted to IEEE Trans. Info. Theory. Download available at http://mars . bell-1abs . com.

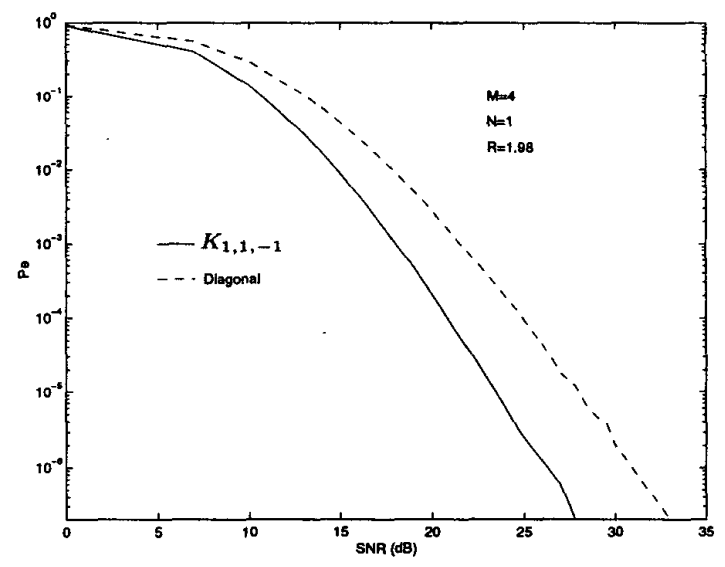

Figure 3: Block-error rate performance of the group $K_{1,1,-1}$ compared with the best diagonal code for $M=4$ transmitter antennas and $N=1$ receiver antenna. The solid line is $K_{1,1,-1}$ having $L=240$ unitary matrices $(R \approx 1.98)$. The dashed line is a diagonal construction with the same rate. 